\title{
Gesetz zur Begrenzung der Managergehälter fällt im Bundesrat durch
}

Der Bundesrat hat das Gesetz zur Kontrolle der Vorstandsvergütung und zur Änderung aktienrechtlicher Vorschriften (VorstKoG) in das Vermittlungsverfahren verwiesen. Die Länder halten die vom Bundestag beschlossene Übertragung der Letztentscheidungsbefugnis über die Vergütungssysteme börsennotierter Gesellschaften auf die Hauptversammlung für nicht geeignet, um exorbitante Managergehälter zu verhindern. Dieses Ziel müsse auf andere Weise erreicht werden, beispielsweise durch die Koppelung der Vorstandsgehälter an das Durchschnittseinkommen der Unternehmensbeschäftigten (BR-Drucks. 637/13 (Beschluss)).

Die derzeit vom Bundestag vorgesehenen Regelungen führen nach Ansicht der Länder unter anderem zu einer unguten Gewichtsverlagerung im sorgfältig austarierten Befugnissystem der drei Organe der Aktiengesellschaft (Hauptversammlung, Aufsichtsrat, Vorstand). Der Aufsichtsrat würde durch die Übertragung der Letztentscheidungsbefugnis über die Vorstandsvergütung auf die Hauptversammlung aufgrund der damit verbundenen Einschränkung seiner Personalhoheit erheblich geschwächt, begründet der Bundesrat seine Entscheidung. Das gelte insbesondere auch gegenüber dem Vorstand, da dem Aufsichtsrat ein wichtiges Druckmit- tel zur Durchsetzung seiner Überwachungsaufgabe entzogen würde.

Das jetzt - zumindest vorerst - gescheiterte Gesetz soll nicht nur das Vergütungssystem, sondern auch das geltende Aktienrecht punktuell weiterentwickeln. Zum Beispiel ist vorgesehen, dass Aktiengesellschaften Kernkapital auch durch die Ausgabe stimmrechtsloser Vorzugsaktien bilden können. Zudem soll das Gesetz die Beteiligungsverhältnisse bei börsennotierten Aktiengesellschaften transparenter machen.

Mehr zum Thema

Sommer, S./Lachmann, M./Judith, A.: Performanceabhängigkeit der Vorstandsvergütung in der Finanz- und Wirtschaftskrise - eine Analyse fixer und variabler Vergütungskomponenten unter Berücksichtigung von Corporate GovernanceMechanismen, in: Welge, M./Witt, P. (Hrsg.): Corporate Governance in mittelständischen Unternehmen, Wiesbaden 2013, S. $89-122$.

SfP $^{*}$ www.springerprofessional.de/4424542

Vera Treitschke, Wiesbaden

\section{Datensicherheit und IT gewinnen bei CFOs an Bedeutung}

Der Datenskandal rund um die angloamerikanischen Spähprogramme verunsichert auch deutsche Finanzvorstände. $\mathrm{Zu}$ diesem Ergebnis kommt das „5. CFO Panel“ der internationalen Anwaltssozietät CMS Hasche Sigle und des FINANCEMagazins im Herbst 2013. Rund 100 CFOs wurden hierbei zu ihrer Einschätzung befragt.

Mehr als ein Drittel der Teilnehmer gab an, dass der Schutz von Unternehmensdaten nicht mehr leicht zu gewährleisten sei. Eine wirklich unsichere oder sehr unsichere Situation sehen aber nur $12 \%$ der CFOs. Fast $40 \%$ der Befragten prüfen Schritte wie Verschlüsselung, Wechsel der Dienstleister oder
Abkehr von Cloud-Lösungen. Die Mehrheit will aber vorerst nicht weiter in Sicherheit investieren.

Auch das Thema IT im Allgemeinen ist in der Prioritätenliste der befragten CFOs in den letzten Monaten gestiegen. Ein Grund hierfür könnte sein, dass Aufsichtsbehörden Datenschutzverstöße inzwischen deutlich aggressiver verfolgen. „Neben dem Risiko einer negativen Öffentlichkeitswirkung besteht für Unternehmen nun zunehmend auch die Gefahr von empfindlichen Geldbußen bei Datenschutzverletzungen“, bestätigt CMS Hasche Sigle-Partner Christian Runte. 